\title{
A Model Study of How Electric Field Structures Affect the Polar Cap $F$ Region
}

\author{
J. J. SOJKA AND R. W. SCHUNK
}

Center for Atmospheric and Space Sciences, Utah State University, Logan

\begin{abstract}
A three-dimensional time-dependent ionospheric model was used to study how electric field structures affect the polar $\boldsymbol{F}$ region. The electric field structures are represented by elongated Volland two-cell models whose dimensions range from tens to $1000 \mathrm{~km}$. These model structures are intended to represent the polar cap electric field for IMF $B_{z}$ northward conditions. A statistical method is used to generate a set of these structures. Their electric field strength and polarity are varied in order to study the $F$ region's dependence on this magnetospheric input. For electric field structures whose size and electric field strengths are consistent with $B_{z}$ northward observations, several ionospheric dependencies were found. The $N_{m} F_{2}$ parameter, in general, decreased due to the presence of the higher electric fields associated with the structures. Decreases ranging from a few percent to a factor of 4 were obtained from the simulations. This decrease in $N_{m} F_{2}$ was further complicated by the initial $N_{m} F_{2}$ conditions, i.e., past history of the flux tube, being quite different when the structure is present. $H_{m} F_{2}$ in the vicinity of a structure can be raised or lowered by up to $100 \mathrm{~km}$, however, this change is not uniquely dependent upon the vertical induced drift. The role of enhanced reaction rates due to elevated ion temperatures is very important. The $F$ region becomes spatially very structured. The modulation of this structure in $N_{m} F_{2}$ is up to an order of magnitude in this study.
\end{abstract}

\section{INTRODUCTION}

Our understanding of the individual physical processes at work in the ionosphere-thermosphere system is good. Both theoretically and experimentally the cause and effect of many of these processes have been studied. Indeed, on a global scale numerical models allow the coupling of these processes to be studied, and the results compared with multi-parameter observations. However, on another level, the degree to which such models simulate the coupling is difficult to judge. Whenever the numerical model gives results not quite consistent with the observations, it is unclear as to whether the model inputs were insufficiently constrained by the observations or whether the model's spatial or temporal resolution was adequate. Hence in general, model-observation discrepancies can only be used with great difficulty and care to infer that the model's physical description is inadequate. Therefore determining how well the global ionospheric-thermospheric models reproduce the overall interactions is extremely difficult; a simple "error analysis" is at best misleading because of its oversimplification. The objective of this study is to determine the global scale ionosphere's response to the "structure" in one of its inputs, i.e., the convection electric field. To date, no quantitative studies have been made to determine the effect of "structure" in global scale ionospheric models.

Since the first high latitude ionospheric model study by Knudsen et al. [1977] several large scale high latitude ionospheric studies have been undertaken [Watkins, 1978; Sojka et al., 1981a; Quegan et al., 1982]. Although the model studies have become more sophisticated over the years, they all follow a similar methodology. They all use globally smooth input functions to represent the neutral atmosphere; neutral wind; and magnetospheric convection, precipitation, and heat flux. Due to the limitations associated with computer time and memory as well as observational coverage of these parameters, this methodology is quite reasonable. However, it is well known that such smooth inputs are the exception rather than the rule. The

\section{Copyright 1988 by the American Geophysical Union.}

models simulate the global system in an averaged sense, and the small scale lengths, on scales of a few to thousands of kilometers, are smoothed away. To date, the effects that such structuring will have on the global simulations has only been handwavingly considered. Each structure is itself a self-consistent physical entity whose detailed characteristics are unknown. The accumulated effect of many structures is difficult to deduce because of the many different time constants involved. Since this study focusses attention on the magnetospheric convection electric field, a few examples from this area will be used to highlight the degree of structure associated with the high latitude electric field.

In the pioneering work of Heppner [1972, 1973, 1977], this very question was extensively addressed. Heppner characterized the smooth electric field signatures using a large data base of OGO-6 polar region electric field measurements. He found several basic patterns and showed how they related to the magnetic activity and the interplanetary magnetic field orientation. However, he also pointed out that on these patterns varying degrees of smaller scale electric field structures were superimposed. Furthermore, because of the abundance and strengths of the smaller scale structures, as many as $50 \%$ of the polar cap crossings could not be characterized unambiguously by a smooth pattern. Figure 11 from Heppner [1977] dramatically demonstrates this problem. More recently, Frank et al. [1986] carried out a multiparameter study of the disturbed polar cap; namely, during periods of theta aurora. Using data from various instruments on the Dynamics Explorer satellites, it was again found that the electric field, as well as other parameters, were highly structured across the polar cap. Figures 5-12 of the Frank et al. paper show structure dominated patterns for each orbit. In addition to these papers, many other in-situ and ground-based observations have been made of the electric field structure.

Table 1 lists pertinent features associated with the structures from a variety of these published observations. The amplitude refers to the departure from the average electric field value while the widths are based on a positive-to-negative peak separation. Both parameters are purely subjective, no detailed numerical analysis has been carried out. From Table 1 it can be seen that the structure range in size from tens of kilometers, (the lowest 
TABLE 1. In Situ Observation of Electric Field Structures

\begin{tabular}{lclcl}
\hline \multicolumn{1}{c}{ Reference } & Figure & Satellite & Amplitude, mV/m & Width, km \\
\hline & 1 & OGO 6 & $10-20$ & $15-500$ \\
Heppner [1972] & 11 & OGO 6 & $5-80$ & $10-800$ \\
Heppner [1977] & 2 & S3-2 & $10-20$ & $30-600$ \\
Burke et al. $[1979]$ & 4 & AE-C & $5-65$ & few-1000 \\
Heelis and Hanson [1980] & 5 & S3-2 & $10-20$ & $40-400$ \\
Smiddy et al. $[1980]$ & $5-9$ & DE-2 & $10-100$ & $10-800$ \\
Frank et al. $[1986)$ & 2 & DE-2 & $15-60$ & few hundred \\
Heelis et al. $[1986]$ & & & & \\
\hline
\end{tabular}

resolution obtainable from these published figures) to $1000 \mathrm{~km}$; their average size being a few hundred kilometers. Also, the electric field variation from the average value ranges from a few millivolts per meter to over a hundred millivolts per meter. For these examples typical electric field variations of 10 to $30 \mathrm{mV} / \mathrm{m}$ were found. Unfortunately, these in situ satellite measurements do not give a direct indication of the structure's two-dimensional form or its duration. To some extent ground-based incoherent radars viewing the polar cap may in the future be able to address this aspect. High resolution electric field observations from the Sondrestrom incoherent scatter radar [Kelly and Vickrey, 1984; Koffman and Wickwar, 1984] and the EISCAT radar system [van Eyken et al., 1984] demonstrate that these radars have the necessary resolution to observe the spatial and temporal properties of local electric field structures.

For these electric field structures to be included in a global scale ionospheric simulation, it is necessary to have a complete spatial and temporal description of the electric field. Unfortunately, no such description is available. Theoretically, our understanding of the processes that lead to these structures is vague, although it is realized that the structures themselves are a complex "auroral-voltage-current" system [Kan and Burke, 1985]. On the smaller scale size (sub-kilometers), treating the plasma structures (irregularities) as plasma turbulence has been reasonably successful [Kintner and Seyler, 1985]. In attempting to describe plasma structures in a global $\boldsymbol{F}$ region model based on the transport formulation, these sub-kilometer irregularities [Kintner and Seyler, 1985] are precluded and, therefore, only structure on scale sizes greater than $10 \mathrm{~km}$ should be considered. The transport formulation does not allow for plasma waves, and, hence, conditions that lead to wave generation cannot be treated. Given that neither an adequate theoretical or observational description of the structures is available, a semiempirical one must be created to carry out the study.

To create a semiempirical model of a polar cap structure, the mutual behavior of the precipitation, electric field, currents and conductivities must be known. At this time no such data base has been studied, although data bases such as the Dynamics Explorer's could well prove adequate. Instead, for simplicity, only one parameter (the electric field structure) will be modeled. As a result the study will not be directly applicable to the ionosphere; however, an indication as to how the model ionosphere quantitatively responds to multiple electric field structures will be obtained. Follow up studies for the other parameters and their interdependence would gradually complete the
picture. Pere.

In section 2 the semiempirical model of a single electric field structure is developed and specific structure polar cap electric field models are described. The results of using these models as an input to the Utah State University (USU) time-dependent ionospheric model (TDIM) are presented in section 3. Section 4 summarizes these results and discusses their implications with respect to present-day "smooth" input simulations.

\section{EleCtric Field StruCture}

\subsection{Model}

In this section a very simple and analytic representation of the polar cap electric field structures is developed. This representation is only weakly based upon theoretical understanding of the structures. The electric field structure is assumed to be associated with a discrete current system into and out of the polar ionosphere. Such polar cap discrete structures are often found to be sun-aligned. Other polar cap features such as "diffuse auroral patches" will not be represented by this numerical simulation. Further the electric field structure is assumed to be associated with finite length parallel sun-aligned sheets of current. The electric field pattern, to zeroth order, is then assumed to be a small elongated Volland two-cell pattern [Volland, 1973, 1975]. In order to quantify this electric field pattern the key parameters are chosen to generate a scale size and electric field amplitude consistent with observations.

A Volland two-cell electric field pattern consists of two regions; a central uniform electric field region which in this case is elliptical in shape, and surrounding this a second region where the electric field diminishes to zero at some outer boundary (again elliptical in shape). The boundary between these two regions is the location of the hypothetical Birkeland current systems. If the two-cell pattern is assumed to be elongated in the sun-aligned direction, then the Birkeland currents are maximized at the dawn-dusk region of the boundaries between the two regions. The appendix shows how the analytic model is constructed.

Each electric field structure is represented by one such twocell pattern. The pattern is defined by a set of parameters: $\left\{x_{0}, y_{0}, r_{0}, f_{\text {in }}, r_{2}, \phi_{0}\right\}$. These parameters represent the following properties:
$\left(x_{0}, y_{0}\right) \quad$ polar cap location at the center of a structure;
$r_{0} \quad$ central region semiminor axis;
$f_{\text {in }}$ ratio of the central regions semimajor axis to its semiminor axis;
$r_{2} \quad$ outermost region semiminor axis (at this boundary the electric field diminishes to zero);
$\phi_{0} \quad$ electric potential drop across the central region.

Given these six parameters the electric field structure can be generated. In the appendix the units associated with these six parameters are defined. 

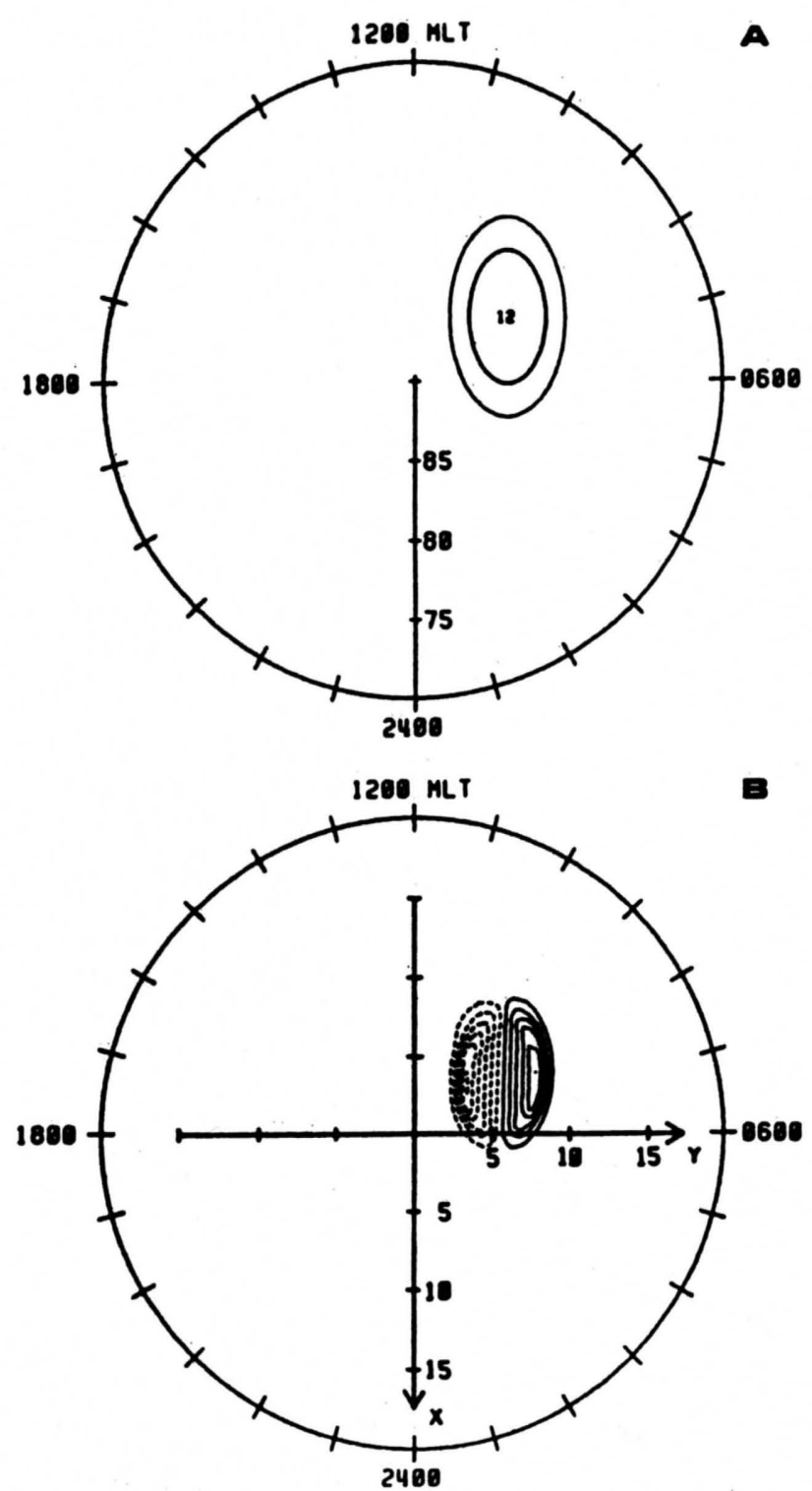

Fig. 1. (a) The area occupied by a modeled electric field structure (large ellipse) and its uniform central region (small ellipse) shown in a magnetic local time (MLT) and magnetic latitude frame. The number inside the small ellipse indicates the dawn-dusk potential across this ellipse in kilovolts. (b) The corresponding electric potential contours drawn at $1 \mathrm{kV}$ intervals shown in the new magnetic $x-y$ coordinate system. Negative potentials are shown as dashed contours. The potential varies from -6 to $6 \mathrm{kV}$ for this electric field structure. The new coordinate system is located at the magnetic pole with the positive $x$ axis lying along the magnetic midnight meridian while the positive $y$ axis lies along the dawn magnetic meridian. Units along each axis correspond to degrees of magnetic colatitude.

At this point polar cap observations of electric field structures have to be used to restrict the values of the six parameters in order to keep the analytic models in reasonable agreement with observations. In the references cited in the introduction it is noted that the structured electric fields are predominantly associated with IMF $B_{z}$ changing sign, zero, or positive (northward). Under $B_{z}$ (southward) conditions and almost irrespective of the activity level the polar cap becomes very quiet and unstructured. Table 1 lists the various electric field data papers used in determining realistic ranges for the electric field structures. For each paper the peak to peak distance, and the range of absolute electric field strengths are tabulated. Since these data were all obtained by in-situ observation from polar cap satellite crossings, they do not give direct information on either the polar cap distribution $\left(x_{0}, y_{0}\right)$ or the elongation of the "two-cell" model parameter $\left(f_{\text {in }}\right)$. Clearly, each parameter is observed to vary from one structure to the next. Therefore these data must be used to generate in a statistical manner the probable distribution or variation for each parameter. The computer model then uses the probability function together with a random number generator to define the various parameters for the electric field structures. The structure's spatial locations $\left(x_{0}, y_{0}\right)$ are distributed evenly over the polar cap while the remaining four parameters are assumed to have a mean value and a Gaussian distribution. A Gaussian random number generator is used for these latter four parameters.

Figure $1 a$ shows the location and dimension of a randomly generated electric field structure. The structure is located in the prenoon sector and has a dawn-dusk central region of $550 \mathrm{~km}$, while the sun-aligned dimension is extended to $920 \mathrm{~km}$. For this structure a total potential drop of $12 \mathrm{kV}$ was selected. Figure $1 b$ shows contours of the electrostatic potential for this electric field model. Contours are drawn at $1 \mathrm{kV}$ intervals with dashed lines representing negative potentials. This pattern is a basic Volland two-cell with ionospheric $F$ region plasma being transported antisunward in the central region. Also shown in Figure $1 b$ is the adopted polar cap $(x, y)$ coordinate system. The positive $x$ axis has its origin at the magnetic pole and lies along the midnight meridian. For both axes the units are degrees of magnetic colatitude. Similarly, the $y$ axis lies along the 0600 MLT magnetic meridian.

\subsection{Adopted Electric Field Models}

For this pilot study the objective is to determine quantitatively to what extent the polar cap electric field structures affect the $F$ region plasma characteristics. To date, global ionospheric simulations have adopted electric field models that do not contain the small scale structures described in the introduction. Hence, for this study two electric field structure models were constructed. They differ by the overall strength of the electric fields associated with their individual structures. A third model will be briefly discussed. It is the reverse polarity of the stronger of the two models. In this light previous global simulations represent a fourth case for which the electric fields associated with the structures are zero. Such a case will be the baseline against which the new simulations will be compared.

The information derived from published polar cap electric field structures (i.e., the contents of Table 1) were used to define the model parameters distributions. These parameters are listed in Table 2 for the first two cases alluded to above. Both cases have identical spatial properties, their individual structures have

TABLE 2. Adopted Mean and Half-Width Values for the Electric Field Structures

\begin{tabular}{lcl}
\hline \multicolumn{1}{c}{ Parameter } & Mean Value & Half-Width \\
\hline & & \\
Central radius $r_{0}$ & $2^{\circ}$ & $1^{\circ}$ \\
Ratio (major axis/minor axis) $f_{0}$ & 1.7 & 0.3 \\
Ratio $r_{2} / r_{0}$ & 1.5 & 0.5 \\
Potential drop case A & $5 \mathrm{kV}$ & $2 \mathrm{kV}$ \\
Potential drop case B & $12 \mathrm{kV}$ & $4 \mathrm{kV}$ \\
\hline
\end{tabular}




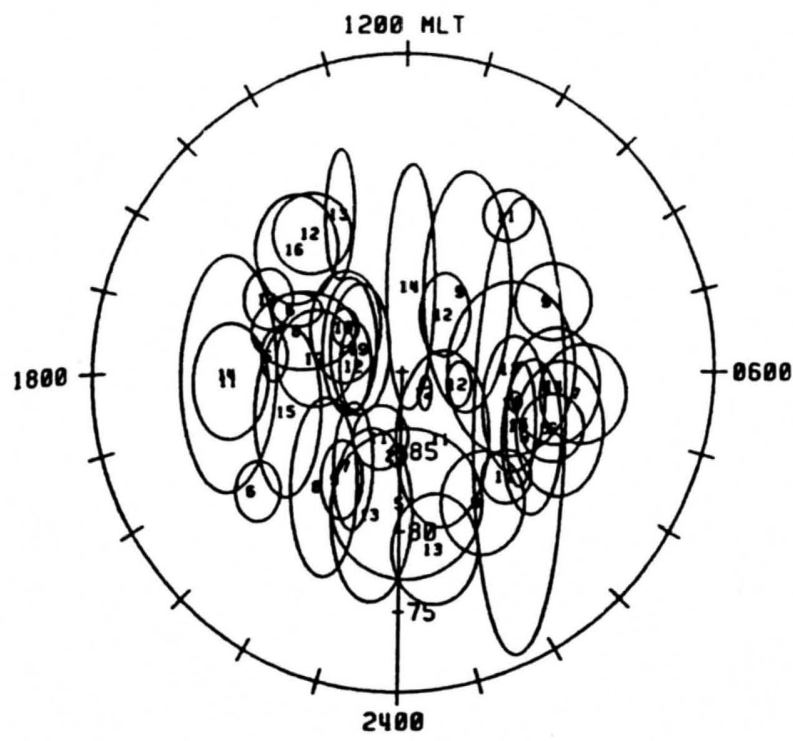

Fig. 2. Distribution of the adopted 45 polar cap electric field structures in the MLT-magnetic latitude frame. Each structure is represented by its uniform central region and labeled in the center with its dawn-dusk potential drop in kilovolts.

a mean central width of $2^{\circ}$ with a Gaussian half width of $1^{\circ} ;$ a mean ratio for the central semi major to semi minor axis of 1.7 with a Gaussian half-width of 0.3 ; and finally a mean outer boundary ratio to the central semiminor axis of 1.5 with a Gaussian half-width of 0.5 .

The case A model has electric potential drops across the structures central regions defined by a mean value of $5 \mathrm{kV}$ and a Gaussian half-width of $2 \mathrm{kV}$, while case $B$ has a $12-\mathrm{kV}$ and $4-\mathrm{kV}$ mean and Gaussian half-width, respectively. In order to complete the electric field structure model, a total of $\mathbf{4 5}$ individual structures were randomly distributed over a $12^{\circ}$ radius polar cap area about the magnetic pole. For both cases $A$ and $B$ the same distribution of parameters for the $\mathbf{4 5}$ structures was adopted; they only differ in their electric field strengths.

The spatial distribution of these structures and their respective central regions is shown in Figure 2 in the MLT-magnetic latitude polar plot. There is considerable overlap between the structures. This will generate rather irregular electric field distributions which will help simulate the observed electric field structure. Inside each central region in Figure 2 there is a number, this corresponds for each structure to the total central region potential drop for the case B model. Figure 3 shows contours of electric potential in the polar MLT-magnetic latitude frame for zero, case A and case B structure models. For these panels the structure electric potentials are added to the reference global scale two-cell high latitude convection pattern. Top panel shows the $K p=3(60 \mathrm{kV})$ global potential pattern. Contours are drawn at $6 \mathrm{kV}$ intervals with negative potentials being represented by dashed contours. The adopted large scale two-cell pattern has a $5^{\circ}$ antisunward offset and exhibits a slight throat pattern in the noon sector [Heelis et al., 1982]. Such ionoction patterns have been extensively used in large scale Roble corote al., 1982; Sojka and Schunk, 1985a]. In Figure 3 the

orotational electric field has not been added into the patterns. The middle panel of Figure 3 shows the case A electric field as these model. Although the contours are no longer as smooth those in the top panel, they do, however, still show predomi- nantly the same overall polar cap pattern. The electric field structure introduced in this panel has only caused distortions in the contours on the 6-kV scale (the contour level). In the lower panel which shows the case B electric potential the situation is quite different. Now the pattern does not resemble that of the top panel. There are many contour "islands." The potential pattern departs significantly from the large scale two-cell pattern in the top panel. Inside the polar region the electric fields associated with the contours are no longer dawn-dusk or uniform.

From these panels it is still difficult to visualize or even compare these electric fields with observations. Figure 4 shows the dawn-dusk electric field for the zero (top panel), case A

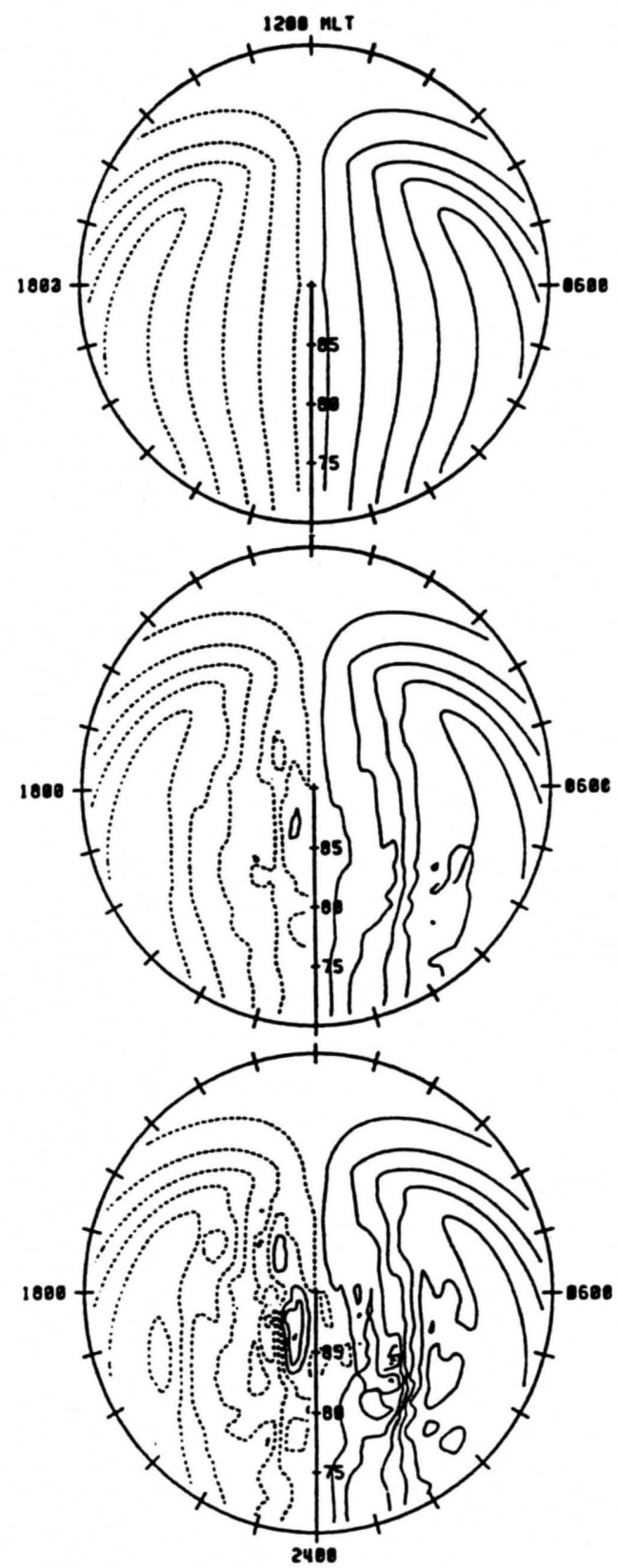

Fig. 3. Polar cap potential contours of the unperturbed two-cell convection pattern (top panel), case A model (middle panel), and case B model (lower panel) shown in the MLT-magnetic latitude frame. Contours are drawn at 6-kV intervals with negative potentials being represented by dashed contours. 

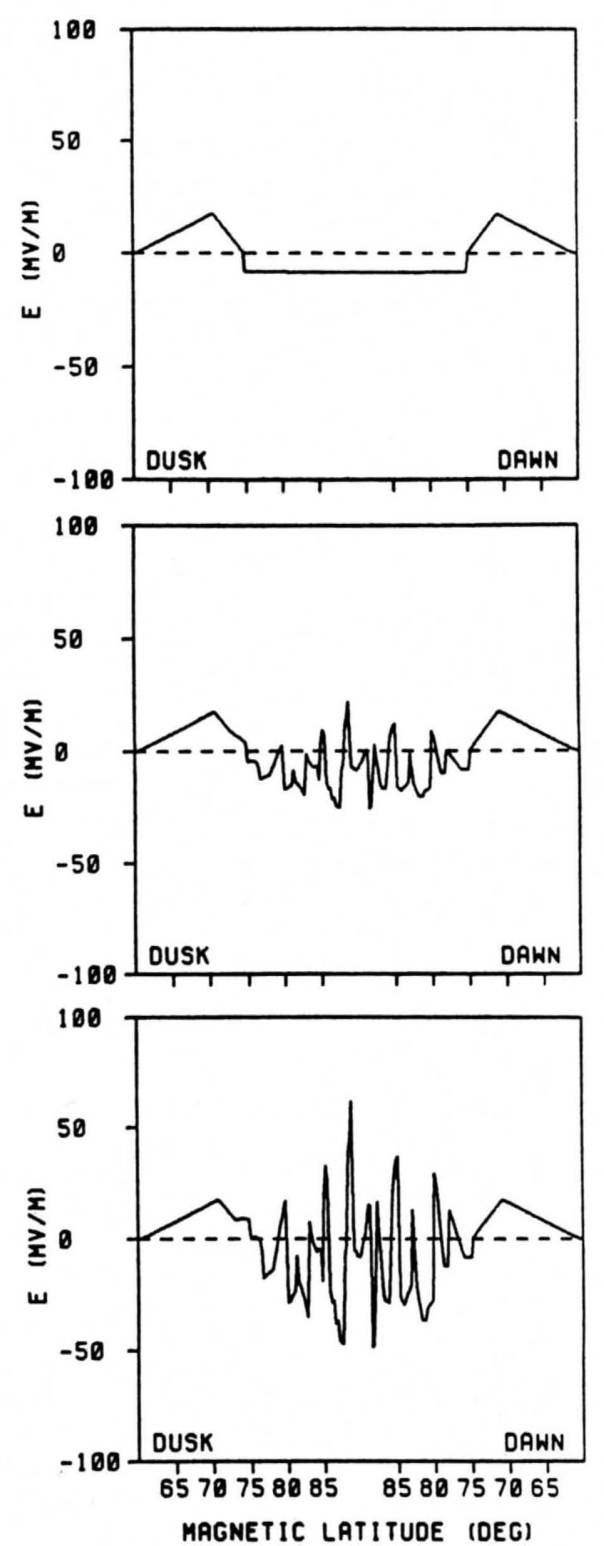

Fig. 4. Electric field variation along the dusk-dawn magnetic meridian for the unperturbed two-cell field (top panel), case A model (middle panel), and case B model (lower panel). The electric field is given in millivolts per meter.

(middle panel), and case B (bottom panel) electric field structure models. The top panel shows the two-cell large-scale electric field which is uniform across the polar cap with a field strength of $\sim 12 \mathrm{mV} / \mathrm{m}$. For case $A$ and $B$ models the polar cap is highly structured. In case $\mathrm{A}$ the electric field strength has a maximum of $25 \mathrm{mV} / \mathrm{m}$ departure from the zero case polar cap electric field. The electric field departure for case $B$ reaches $70 \mathrm{mV} / \mathrm{m}$ for one structure although more typically the departure is on the order of $40 \mathrm{mV} / \mathrm{m}$. These electric field strengths are quite consistent with the data given in Table 1. Furthermore, the spatial scale of these structures ranges from about $1^{\circ}$ to $5^{\circ}(110$ to $550 \mathrm{~km}$ at $F$ region altitudes) along the dawn-dusk meridian. The spatial scale sizes are also consistent with the information in Table 1. Unfortunately, at this time no detailed observational information is available on the spatial scales in a direction orthogonal to that shown in Figure 4. The model assumes these dimensions to be extended by about a factor of 1.7 over those in the dawn-dusk direction.

One of the major functions of the electric field in the $F$ region is to drive plasma convection. The convection paths followed by the plasma are $\left(\mathbf{E}_{t} \times \mathbf{B}\right) / B^{2}$ drift paths; where the electric field $\mathbf{E}_{t}$ is the vector sum of the electric field described above with the corotational electric field, and B is the magnetic field. Figure 5 shows eight selected plasma convection trajectories for the zero (top panel), case A (middle panel), and case B (bottom panel) electric field structure models summed with the corotational
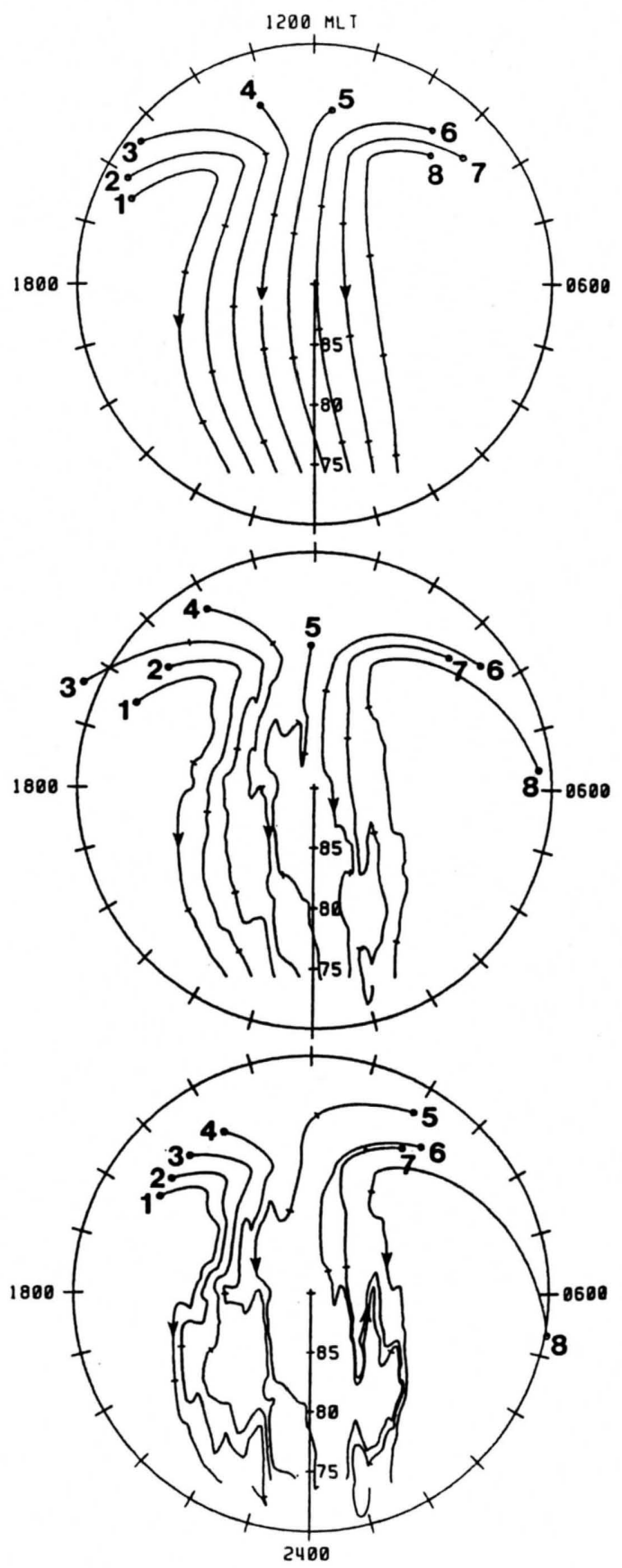

Fig. 5. Plasma convection trajectories for two-cell unperturbed study, (top panel), for case A model (middle panel), and for case B model (lower panel) shown in the MLT-magnetic latitude frame. Circles denote the start location for each trajectory while tick marks indicate one hourly time intervals. 
TABLE 3. Convection Times in Seconds From $x=-5.5$ to $x=15.5$

\begin{tabular}{cccc} 
Trajectory & Zero Case & Case A & Case B \\
\hline 1 & 5898 & 6540 & 3439 \\
2 & 6187 & 5583 & 5370 \\
3 & 6505 & 5603 & 3650 \\
4 & 6853 & 4940 & 3697 \\
5 & 7242 & 3815 & 2008 \\
6 & 7686 & 6055 & 6486 \\
7 & 8201 & 9742 & 5771 \\
8 & 8812 & 3660 & 2276 \\
\hline
\end{tabular}

electric field. The eight trajectories have been selected such that they all end equally spaced along the $x=16$ line at $80,000 \mathrm{~s}$ UT. This is most clearly seen in the top panel where all eight trajectories end in a line. Each trajectory end point is $2^{\circ}$ apart along the $x=16$ line of the coordinate system defined in Figure $1 b$. Such a choice of end points is somewhat arbitrary, but it would correspond to points along an approximate satellite track or lie inside the field of view of an azimuthally scanning incoherent scatter radar. In both cases the data are all collected over a relatively short period of time, a few minutes at most. Having spatially and temporarily synchronized the trajectory end points, all earlier trajectory points are no longer synchronized between the three models. This being a direct consequence of the three different electric field models leading to different convection paths and speeds. For each of the three cases the trajectories are started outside the polar cap and where possible in sunlight. The final UT of 80,000 s was chosen because under the $K p=3$, two-cell convection pattern, top panel Figure 5, the starting points are all in sunlight giving reasonably uniform starting density conditions. Once these trajectories enter the polar cap they also enter darkness, the conditions which are most sensitive to stimulated density changes caused by the $\left(\mathbf{E}_{t} \times \mathbf{X} \quad \mathbf{B}\right) /$ $B^{2}$ drift mechanisms. Tick marks along the trajectory indicate hourly intervals. The trajectories take $\sim 2 \frac{1}{2}$ hours to convect across the polar cap.

In the middle and lower panels of Figure 5, the net polar cap convection is still antisunward although there are marked regions of sunward convection. The tick marks drawn at hourly UT intervals indicate that the transpolar cap times are changing significantly. Indeed this change has caused some of the dayside start locations to lie in darkness (due to the change in universal time in this region). To avoid this the trajectory has been followed to earlier times when it was located in the morning sector auroral oval. Trajectory 8 suffers from this problem in both the case $A$ and $B$ models.

The transpolar convection time differences are highlighted in Table 3 , in which the time each trajectory takes to go from the $x$ $=-5.5$ location to the $x=15.5$ ( $\approx$ endpoint) is tabulated. These two locations are $\pm 10^{\circ}$ from the center of the unstructured convection pattern, which has an antisunward offset of $+5^{\circ}$. For the zero structured case, the times range systematically from 5898 seconds to 8812 seconds. These increasing times represent the effect of the corotational field in conjunction with the constant two-cell polar cap electric field. Case A times vary randomly compared to the zero case, and with the exception of trajectories 1 and 7 are all shorter than the corresponding zero tre trajectory. In the B case, the times are all shorter, with thectories 5 and 8 being about one quarter of the correspondcase times. The 30-min transport times correspond to an average convection speed of $1.2 \mathrm{~km} / \mathrm{s}$. In going from no structures to case $\mathrm{A}$ the time changes appear larger than going from case $A$ to case $B$ even though for the latter comparison the structure electric fields have approximately doubled. This is a consequence of both the case A and B structures having the same area, and hence the overall effect of changing just the potential is not as significant as changing the area and the potential. The modified transit times not only depend on the number and magnitude of the electric field structures, but also upon the area occupied by the structures. These faster transport times are expected to have significant implications for the polar cap density distributions. Naively, one could be tempted to infer higher densities along the faster moving trajectories because inside the polar cap the plasma is in darkness and decaying (i.e., reducing the time spent in darkness reduces the amount of decay). However, as the next section will show, the situation is considerably more complex even in the absence of plasma production sources.

\section{MODELED IONOSPHERIC PARAMETERS}

The three electric fields described in the previous section were the variable inputs for three runs of the USU time-dependent ionospheric model (TDIM). This model and its history are described in section 3.1. Additional input parameters must be specified before the TDIM can be run; these are defined in section 3.2. The results of running the TDIM for each of the three electric field cases are given in subsections 3.3. and 3.4.

\subsection{Ionosphere Model}

The ionospheric model was initially developed as a midlatitude, multiion $\left(\mathrm{NO}^{+}, \mathrm{O}_{2}^{+}, \mathrm{N}_{2}^{+}\right.$, and $\mathrm{O}^{+}$) model by Schunk and Walker [1973]. The time-dependent ion continuity and momentum equations were solved as a function of altitude for a corotating plasma flux tube including diurnal variations and all relevant $E$ and $F$ region processes. This model was extended to include high-latitude effects due to convection electric fields and particle precipitation by Schunk et al. [1975, 1976]. A simplified ion energy equation was also added, which was based on the assumption that local heating and cooling processes dominate (valid below $500 \mathrm{~km}$ ). Flux tubes of plasma were followed as they moved in response to convection electric fields. A further extension of the model to include the minor ions $\mathrm{N}^{+}$and $\mathrm{He}^{+}$, an updated photochemical scheme, and the MSIS atmospheric model is described in Schunk and Raitt [1980].

The addition of plasma convection and particle precipitation models is described in Sojka et al. [1981a, b]. More recently, the ionospheric model has been extended by Schunk and Sojka [1982a] to include ion thermal conduction and diffusionthermal heat flow, so that the ion temperature is now rigorously calculated at all altitudes between $120-1000 \mathrm{~km}$. The adopted ion energy equation and conductivities are those given by Conrad and Schunk [1979].

\subsection{Additional TDIM Inputs}

Even though for this study attention is focused on the convection models, a number of other parameters, which are inputs to the ionospheric model, need to be described. In solving the equations for the ions listed above, a lower $(120 \mathrm{~km})$ boundary condition of chemical equilibrium and an upper $(800 \mathrm{~km})$ boundary condition of zero flux were imposed. The solar EUV and neutral atmosphere (MSIS) were specified by choosing the following solar and seasonal conditions; 1981 day 321, solar maximum, winter. Specifically, an $A p$ of 30 and $F 10.7$ of 170 


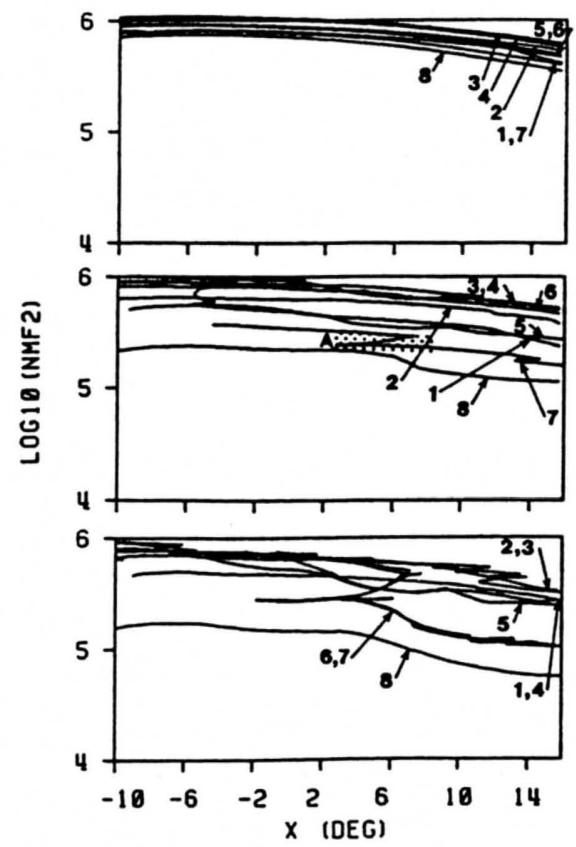

Fig. 6. $N_{m} F_{2}$ densities for each of the trajectories shown in Figure 5 plotted as a function of the flux tubes' $x$ location. The panels correspond to the unperturbed two-cell, case A model, and case B model electric fields in the top, middle, and lower panels, respectively. The density is plotted as a logarithm to the base 10 of the density $\left(\mathrm{cm}^{-3}\right)$.

were adopted for the studies. In addition, a simplistic neutral wind flowing antisunward over the polar cap with a speed of 200 $\mathrm{m} / \mathrm{s}$ was used. This wind pattern is described by Sojka et al. $[1981 a, b]$.

At high latitudes the other main magnetospheric input is the auroral oval, which acts both as a plasma production and heat source. The adopted auroral pattern for the case of two-cell convection $(K p \approx 3)$ was the conventional Spiro et al. [1982] auroral energy flux model for an $A E$ value of 400 . Strongest auroral precipitation is present in the night sector oval with energy fluxes reaching $1.6 \mathrm{erg} \mathrm{cm}^{-2} \mathrm{~s}^{-1}$. These regions of high energy fluxes all lie outside of the polar cap region and therefore do not significantly affect this study.

By using the ionospheric model, the various inputs, and the convection cases described in section 2.2 , three specific ionospheric density studies were made. For each run the eight trajectories shown in Figure 5 were followed over the polar cap as described. Each study kept the solar thermospheric and magnetospheric inputs fixed for the entire simulation period, except for solar and thermospheric UT variations. In the following sections the three studies will be contrasted for a variety of ionospheric parameters.

\section{3. $N_{m} F_{2}$}

Electron density profiles were obtained from the TDIM every few seconds (typically 5-30 s) along each trajectory. As stated earlier each trajectory begins where possible with the appropriate sunlight steady state profile. Displaying data from the eight trajectories for the three models is somewhat difficult, since the spatial coordinates and UT are no longer readily correlated between the runs. In fact for the selected trajectories only the end points have a correlation, i.e., by choice they all end at $80,000 \mathrm{~s}$ UT at fixed locations. Because the aim of the study is to look quantitatively at the effects of the structured $E$ fields on the ionospheric plasma parameters, the normal method of presenting the global data, i.e., two-dimensional polar contour or color plots of specific parameters at a fixed altitude, is not useful because of its qualitative nature. Instead the parameters will be plotted directly as a function of the trajectory $x$ location across the polar cap. In doing so the details of how each structure in the electric field affects the ionosphere can be seen.

Figure 6 shows how the $F$ region peak electron density $\left(N_{m} F_{2}\right)$ varies along each trajectory for the zero (top panel), case $\mathrm{A}$ (middle panel), and case B (bottom panel) model simulations. The densities are plotted against the trajectory $x$ location. This being the location along the noon-midnight meridian direction, see Figure $1 b$ for the definition of the axis. In the top panel where the plasma convection is primarily antisunward and uniform (see Figure 5 top panel), the trajectories move from the most sunward location, -10 , over the pole to a final night side location of +5.5 . During this transpolar crossing the plasma moves into darkness with no particle precipitation. Therefore the plasma density is expected to decrease due to dominance of plasma recombination processes. Indeed, this is observed; over this distance $N_{m} F_{2}$ is found to decrease by about a factor of 2 . The trajectories begin with a range of $N_{m} F_{2}$ values which correspond to the different solar conditions at which each trajectory begins (see Figure 5 top panel). To zeroth order this spread is maintained as the plasma flux tubes convect over the polar cap. At a higher resolution it is found that the final relative densities change by a few tens of percent. This latter difference is attributed to the slightly different transpolar times for these eight trajectories (see Table 3).

Once the electric field becomes structured the systematic trends discussed above disappear; middle and bottom panels show this graphically. In the middle panel, which corresponds to $N_{m} F_{2}$ along the eight trajectories shown in Figure 5 (middle panel), the densities show more than a factor of 4 difference between each other. The relative difference from $x=-10$ to $x=+15.5$ does not remain constant, and the initial densities are not the same as in the top panel. Although the plasma still converts in a net antisunward direction, there are places where some of the trajectories skirt round electric field structures, see Figure 5, middle panel. In Figure 6, middle panel, these loops which include sunward excursions are represented by the $N_{m} F_{2}$ curve becoming multi valued as a function of $x$. The shaded area labeled $\boldsymbol{A}$ highlights such a region.

Clearly, the differences in density at the final locations for case $A$ are significantly larger than those for the zero case. In all eight trajectories the case A final densities are lower than or equal to the corresponding zero case densities. What are the sources of these differences? Two mechanisms are primarily responsible and both stem from the presence of the electric field structures. First, due to the modified transpolar convection speeds the trajectories all begin on the sunward side of the polar cap at different universal times and spatial locations. This results in quite different initial densities. Indeed two of the trajectories do not reach -10 on the $x$ axis, see Figure 5 middle panel. Furthermore, the trajectory labeled 8 , which corresponds to trajectory 8 no longer reaches sunlight on the dayside, hence its initial density is completely different from trajectory 8 in the zero case (top panel). In fact, the initial density for this trajectory is based upon the assumption that the trajectory has just left the auroral oval and that a steady state at the edge of the oval is equivalent to its earlier history. Clearly, no verification of this is possible without an extensive auroral precipitation and convec- 
TABLE 4. Final $N_{m} F_{2}$ Values

\begin{tabular}{clll}
\hline Trajectory & Zero Case & Case A & Case B \\
\hline 1 & $4.27 E 5^{*}$ & $2.40 E 5$ & $2.75 E 5$ \\
2 & $5.37 E 5$ & $3.89 E 5$ & $3.16 E 5$ \\
3 & $6.31 E 5$ & $5.13 E 5$ & $3.31 E 5$ \\
4 & $5.89 E 5$ & $5.25 E 5$ & $2.69 E 5$ \\
5 & $5.01 E 5$ & $2.75 E 5$ & $2.45 E 5$ \\
6 & $4.68 E 5$ & $4.68 E 5$ & $1.07 E 5$ \\
7 & $4.07 E 5$ & $1.62 E 5$ & $1.02 E 5$ \\
8 & $3.63 E 5$ & $1.15 E 5$ & $0.56 E 5$ \\
\hline
\end{tabular}

$* 4.27 E 5=4.27 \times 10^{5} \mathrm{~cm}^{-3}$.

tion analysis. The consequence is that such trajectories can have a wide range of initial conditions that would depend upon their past history in the dark auroral oval.

The second cause of the enhanced density decrease is more directly associated with the electric field structure. In skirting round an electric field structure the $N_{m} F_{2}$ value shows a marked decay; see shaded area in Figure 6 middle panel. Even more marked density reductions are to be seen in the lower panel, the $B$ case where the electric field structures are stronger. In subsection 3.2.3, the details of this enhanced interaction will be studied.

Since only eight trajectories have been studied for each case, it is exceedingly difficult to deduce statistically significant trends. Because of the large number of parameters that are involved in defining the structure electric fields, and then using random number generators to individually select these parameters, this study is not extensive enough to be treated as a Monte Carlo simulation. Hence this study can only indicate the type of trends associated with the structured electric fields.

Table 4 highlights the differences in $N_{m} F_{2}$ at the trajectory end points for the 3 cases. From Figure 6 it is evident that $N_{m} F_{2}$ depends sensitively on the structured electric fields. For all eight trajectories of case $\mathbf{A}$, the densities at the end of the trajectories were lower than or equal to the corresponding zero case. With one exception (trajectory 1), the case B densities were lower than the corresponding case $\mathrm{A}$ densities.

The flux tube transit times for these trajectories also show similar trends. Six of the case A trajectories are shorter than the corresponding zero case, while seven of the case B trajectories are shorter than the corresponding case A trajectories. As an indication of the stochastic nature of the undersampled simulation, the exceptional trajectories in these two comparisons are not correlated, i.e., the slower case $B$ trajectory is not the higher density trajectory. This is further highlighted by the final density differences in Table 4. The differences in final $N_{m} F_{2}$ between case $A$ and the zero case range from being the same (trajectory 6), to being 3.2 times lower. Likewise, the difference between case $B$ and case $A$ extends from being 1.14 times larger to a factor of 4.3 times lower. However, when the $B$ case is compared to the zeroth case for the eight trajectories all are between a factor of 1.6 to 6.5 times lower. For this limited study, these specific factors can only be used to give a zeroth-order indication of the $N_{m} F_{2}$ trends associated with the multiple electric field structures.

3.4. $H_{m} F_{2}$ Associated with the ionospheric $N_{m} F_{2}$ is the peak altitude,
$h_{m} F_{2}$. Figure 7 shows the variation of $h_{m} F_{2}$ as a function of $x$ for the zero (top panel), case A (middle panel), and case B (bottom panel) electric field models. Each panel shows all eight trajectories. The layout of this figure is the same as for Figure 6. All eight zero case trajectories have to within a few kilometers the same $h_{m} F_{2}$ variation, decreasing from $\sim 360$ to $310 \mathrm{~km}$. For the case A (middle panel) and even more so for the case B (bottom panel) $h_{m} F_{2}$ varies significantly between the eight trajectories. In case $\mathrm{B}, h_{m} F_{2}$ approaches $500 \mathrm{~km}$ for several of the trajectories. The $h_{m} F_{2}$ spread for case A is about $50 \mathrm{~km}$ while for case B it extends to $150 \mathrm{~km}$. These large $h_{m} F_{2}$ departures are associated with the more intense electric field structures in which a vertical induced drift $\left(V_{\|}\right)$leads to $h_{m} F_{2}$ being raised or lowered.

\subsection{Influence of $\mathbf{E}$}

Figure 8 shows $N_{m} F_{2}, h_{m} F_{2}, V_{\|}$, and $E_{\perp}$ along trajectory 7 for the case A electric field study. These four parameters are plotted against time, in hours, from the start of this trajectory. In this manner the time spent by the plasma flux tube in each structure is evident. Two events have been highlighted by shading and are labeled $\mathrm{A}$ and $\mathrm{B}$. These two events lead to major changes in $h_{m} F_{2}$ and to a lesser extent $N_{m} F_{2}$.

The two lower panels in Figure 8 show details of the electric field structures. $E_{\perp}$ is a measure of the horizontal electric field strength which not only leads to the plasma convection speed but also ion heating via enhanced ion neutral collisions as the electric field increases [see Schunk et al., 1975, 1976]. The electric field peaks at $\sim 100 \mathrm{mV} / \mathrm{m}$ in event $\mathrm{A}$. For this trajectory the average electric field is about $35 \mathrm{mV} / \mathrm{m}$, which is significantly larger than the zero case value of $\sim 12 \mathrm{mV} / \mathrm{m}$. In the panel above the bottom panel, the vertical induced drift due to the electric field is shown, upward drifts being positive. These vertical drifts range from $80 \mathrm{~m} / \mathrm{s}$ (upward) to $-75 \mathrm{~m} / \mathrm{s}$ (downward). The effect of the strong upward drifts in events $A$ and $B$ is clearly evident on $h_{m} F_{2}$ (third panel from the bottom). $H_{m} F_{2}$ rapidly increases as $V_{\|}$increases. Event A leads to an $h_{m} F_{2}$ close to $500 \mathrm{~km}$.

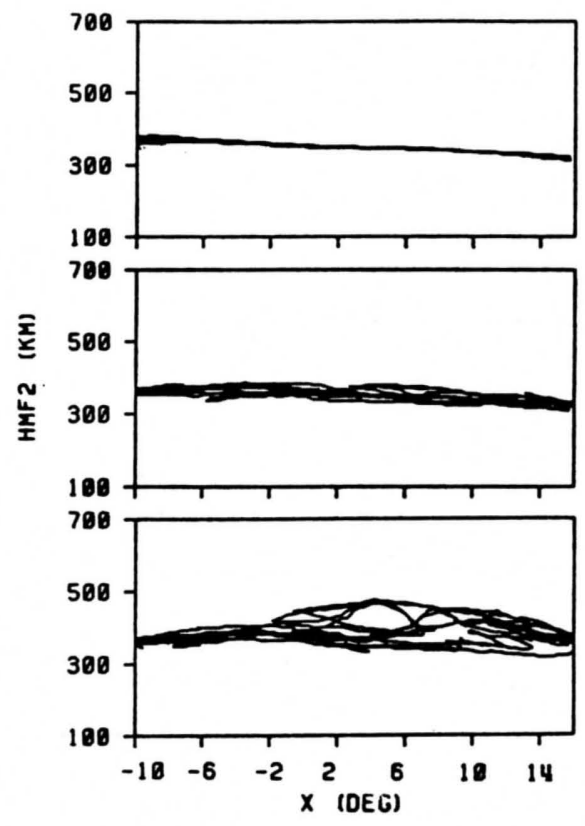

Fig. 7. $H_{m} F_{2}$ altitudes for each of the trajectories shown in Figure 5 plotted as a function of the flux tubes' $x$ location. The panels correspond to the unperturbed two-cell, case A model, and case B model electric fields in the top, middle, and lower panels, respectively. 


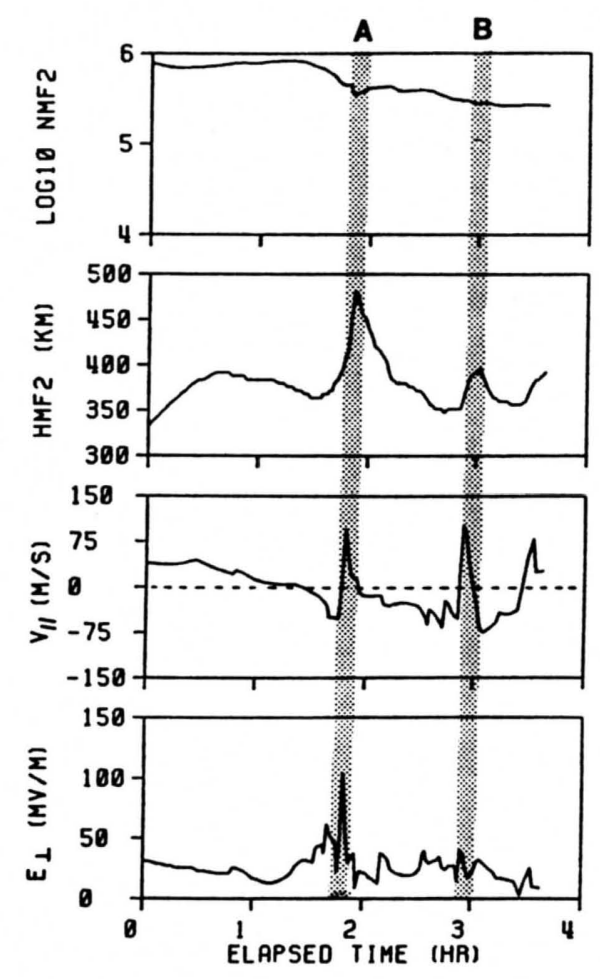

Fig. 8. Variation of the $N_{m} F_{2}$ (top panel), $h_{m} F_{2}$ (upper middle panel), induced upward drift (lower middle panel), and horizontal electric field magnitude (bottom panel) along trajectory 7 for the case A model electric field. The data are plotted as a function of elapsed time along this trajectory. Shading is used to highlight the two times when this trajectory passes through marked fluctuations in the induced vertical drift; the first event is labeled $A$ and the second $B$.

Following the rapid increase in both $V_{\|}$and $h_{m} F_{2}$ the recovery is significantly slower for $h_{m} F_{2}$; tens of minutes compared to the rise time of a few minutes for $V_{\|}$.

$N_{m} F_{2}$ (top panel) does not show as marked a signature during events $\mathrm{A}$ and B. Initially in event $\mathrm{A}, N_{m} F_{2}$ drops by about $25 \%$ but then recovers somewhat, while in event B $N_{m} F_{2}$ shows a negligible response. In contrast $N_{m} F_{2}$ shows the most marked decrease in the 20-min period prior to event A. During this period $N_{m} F_{2}$ is decreased by a factor of more than 2, also in this period the average electric field is $\sim 50 \mathrm{mV} / \mathrm{m}$. This larger electric field acting for $\sim 20 \mathrm{~min}$ is able to maintain a high ion temperature which maintains a rapid conversion of $\mathrm{O}^{+} \rightarrow \mathrm{NO}^{+}$ and, consequently, a fast recombination rate [see Schunk et al., $1975,1976]$. As a result of this enhanced chemical reaction the $F$ region topside profile is being modified by a process other than diffusion. Indeed in Figure 8 immediately prior to event $A$, $h_{m} F_{2}$ is increasing even although $V_{\|}$is downward. The compositional changes in this location are dominant over the induced vertical downward drift.

Another very important point to be drawn from Figure 8 is how complex and apparently uncorrelated are the changes in $N_{m} F_{2}$ and $h_{m} F_{2}$. This is the case for a very restricted theoretical simulation in which only one input is allowed to vary. The overall changes in $N_{m} F_{2}$ do not reflect the high degree of structure in the electric field. $N_{m} F_{2}$ and to a lesser extent $h_{m} F_{2}$ appear to filter out the marked variations on the seconds to a few minutes time scale. If $N_{m} F_{2}$ is looked at with higher resolution, it does respond at the few percent level to some of these changes. $N_{m} F_{2}$ can respond relatively rapidly to only a small degree via the energy equation, where the electron and ion temperature time constants are a few seconds and tens of seconds, respectively.

\subsection{Ion Composition}

As alluded to in the previous subsection, the $F$ region ion composition is radically altered in the electric field structure. This composition change arises from the enhanced rate of the $0^{+}$ $\rightarrow \mathrm{NO}^{+}$reaction in the region of elevated ion temperature, itself caused by the enhanced electric field. In Figure 8 the shaded region referred to as event A shows variations of $N_{m} F_{2}$ and $h_{m} F_{2}$ which are not simply described by transport and diffusion processes. This event is looked at with higher resolution as a series of altitude profiles of both $\mathrm{O}^{+}$and the molecular ions in Figure 9 . Both panels show five sets of profiles, the molecular profiles are labeled 1 to 5 . Corresponding pairs of $\mathrm{O}^{+}$and molecular profiles have the same line style. Ion density in $\mathrm{cm}^{-3}$ is plotted logarithmically to the base 10 against altitude from 120 to $800 \mathrm{~km}$.

In panel A the five pairs of curves represent a time sequence spanning $95 \mathrm{~s}$ starting with profile 1 and ending with profile 5 . During this time the electric field is rapidly increasing from 29 $\mathrm{mV} / \mathrm{m}$ to $90 \mathrm{mV} / \mathrm{m}$ (see Figure 8 event A). Paralleling this electric field increase is a corresponding ion temperature $\left(T_{i}\right)$ increase from 1350 to $5000^{\circ} \mathrm{K}$ at $800 \mathrm{~km}$. This electric field has generated a $T_{i}$ hot spot [Schunk and Sojka, 1982b]. As a result, the reaction rate between $\mathrm{O}^{+}$and $\mathrm{NO}^{+}$is enhanced causing $\mathrm{O}^{+}$to be converted to $\mathrm{NO}^{+}$. Hence the molecular ion profile which is primarily $\mathrm{NO}^{+}$at high altitudes is rapidly enhanced. This enhancement is highlighted by the large shaded arrow. $\mathrm{NO}^{+}$at high altitudes $(>350 \mathrm{~km})$ is enhanced by $1-3$ orders of magnitude. At $800 \mathrm{~km}$ the molecular ion density has increased from 3 to $\sim 100 \mathrm{~cm}^{-3}$. Also the height of the molecular peak has increased from 240 to $305 \mathrm{~km}$. During this time $\mathrm{O}^{+}$is also being modified causing the bottomside to be rapidly eroded and effectively lifted by $50 \mathrm{~km}$, and the small arrow indicates this trend. The $\mathrm{O}^{+}$ peak is also being eroded away which causes it to appear to be lifting. This lifting of the peak and reduction in density is further enhanced by the increased $T_{i}$, which increases the scale height. $H_{m} F_{2}$ is also enhanced by the strong upward induced drift in event A (see Figure 8). As the $\mathrm{O}^{+}$peak is raised from 380 to $\sim 480 \mathrm{~km}$, its density is decreased by about $25 \%$.

In panel B (Figure 9) the subsequent "recovery" part of event $A$ is shown. The electric field decreases from $90 \mathrm{mV} / \mathrm{m}$ to $35 \mathrm{mV} / \mathrm{m}$ in a $220 \mathrm{~s}$ period. Again, the curve labeled 1 represents the start of this period, while curve 5 corresponds to the end of this period. During this period the $T_{i}$ and $V_{\|}$are decreasing leading to a lowering of the peak. As $T_{i}$ decreases the scale height reduces and this leads to a slight enhancement of the $0^{+}$ peak, in fact in $N_{m} F_{2}$. The molecular ions no longer experiencing the favorable production from $\mathrm{O}^{+}$, recombine in order to get back to what is primarily chemical equilibrium appropriate to the $T_{i}$. Consequently, molecular ion density decreases and the peak is lowered. However, the time constant is somewhat slower for this process of recombination compared to the rapid $\mathrm{O}^{+}-$ $\mathrm{NO}^{+}$production process. In the $3^{\frac{1}{2}} \mathrm{~min}$ period the molecular density above $350 \mathrm{~km}$ has only decreased by $\sim 1^{1 / 2}$ orders of magnitude. The decrease takes about four times longer than the original increase.

An additional feature associated with the electric field structure is shown in Figure 9. At high altitudes $(800 \mathrm{~km})$ the molectlar ion density is normally very low, typically less than $10 \mathrm{~cm}^{-3}$ (see curve 1 in Figure 9 panel A). However, during the event, in 
A

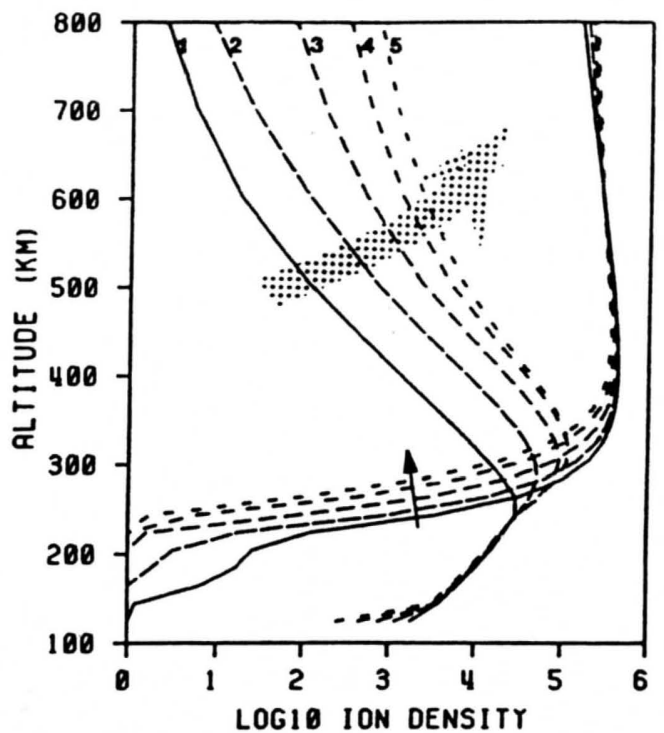

B

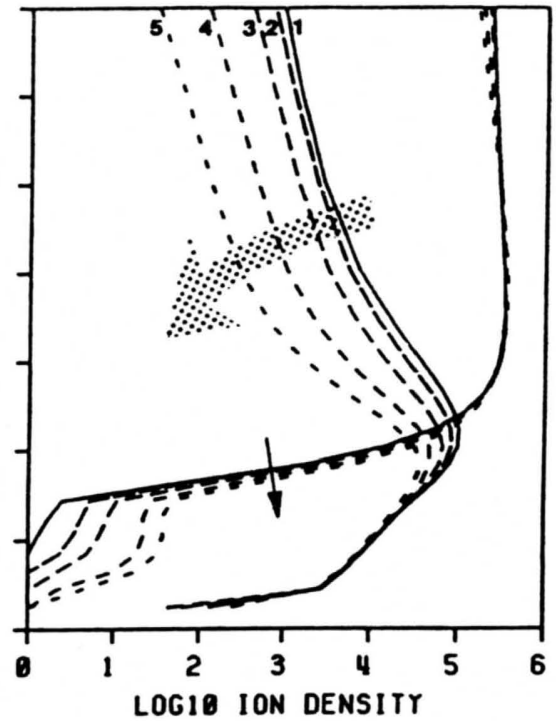

Fig. 9. Variation of the $\mathrm{O}^{+}$and molecular ion density profiles for event $\mathrm{A}$ in Figure 8. The left panel corresponds to increasing disturbance while the right panel corresponds to the disturbance decaying. Unlabeled profiles represent $\mathrm{O}^{+}$while the profiles labeled 1 to 5 are for the molecular ions. The shaded arrows indicate how the topside molecular ion abundance is changing with time, and the arrows show how the bottom side $\mathrm{O}^{+}$density varies with time.

this case a period of about 4 minutes for the plasma flux tube to move through this structure, the molecular ion density is considerably enhanced. The electric field structure acts as a pump to drive molecular ions out of the bottomside ionosphere. It also acts as a heater. The topside ion temperature is raised to $\sim 5000^{\circ}$ $\mathrm{K}$ in event A shown in Figures 8 and 9.

\subsection{Polarity of the Electric Field Structures}

In setting up the electric field structure model, all 45 individual structures in both cases $A$ and $B$ were given a dawn-dusk electric field in their central regions. This choice produced antisunward convection through the center of each cell. Alternatively, it can be argued that during periods of northward IMF these polar cap structures give rise to sunward convection in their central region. Since no clear understanding of these structures is currently available, this latter situation would seem just as plausible as the assumed model of a structure. The question then is how sensitive is this study to the polarity of the electric field structures. To study this a further model case was computed. This was a repeat of case B with all 45 electric field structures having a dusk-dawn orientated central region electric field. For this reverse case, the structures central regions would tend to cause the plasma to convect sunward. The magnitudes of these electric fields would be identical to those shown in Figure 4 , bottom panel. However, in this case the electric field polarity Would be reversed, about a value of $-12 \mathrm{mVm}^{-1}$, which is the electric field in the polar cap for the two-cell pattern.

Figure 10 shows the variation of $N_{m} F_{2}$ (top panel) and $h_{m} F_{2}$ (bottom panel) for all eight trajectories in the same format as the case B $N_{m} F_{2}$ and $h_{m} F_{2}$ shown in Figures 6 and 7 (bottom panels), respectively. Although each trajectory leads to a different value of $N_{m} F_{2}$ compared with the case B model, both have the same overall trend. The endpoint densities vary over the same range, reduced by a few percent to a factor $\sim 8$ of the density expected in the zero case. $H_{m} F_{2}$ undergoes changes which are also similar to those described for case B. It is enhanced up to almost 500 $\mathrm{km}$. Overall, the result of reversing the polarity of the electric field structures is not significantly different to the plasma trends obtained for the original dawn-dusk orientated electric field structures. Specific details such as trajectory start locations, the plasma path through a structure, or where strong electric fields are experienced are quite different.

\section{SUMMARY}

In this paper the $F$ region's response (as represented by the TDIM) to a series of electric field structures was studied theoretically. The individual electric field structures were modeled as elongated two-cell Volland patterns. Their dimensions were small compared to the large scale high latitude convection pat-
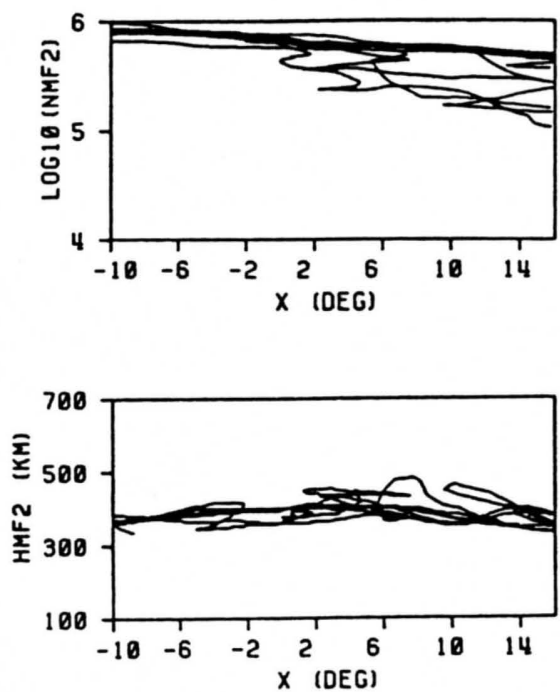

Fig. 10. $N_{m} F_{2}$ (top panel) and $h_{m} F_{2}$ (lower panel) variations along the eight trajectories associated with the reverse case $B$ model electric field plotted as a function of the flux tubes' $x$ position. The density is plotted as the logarithm base 10 of the density $\left(\mathrm{cm}^{-3}\right)$. 


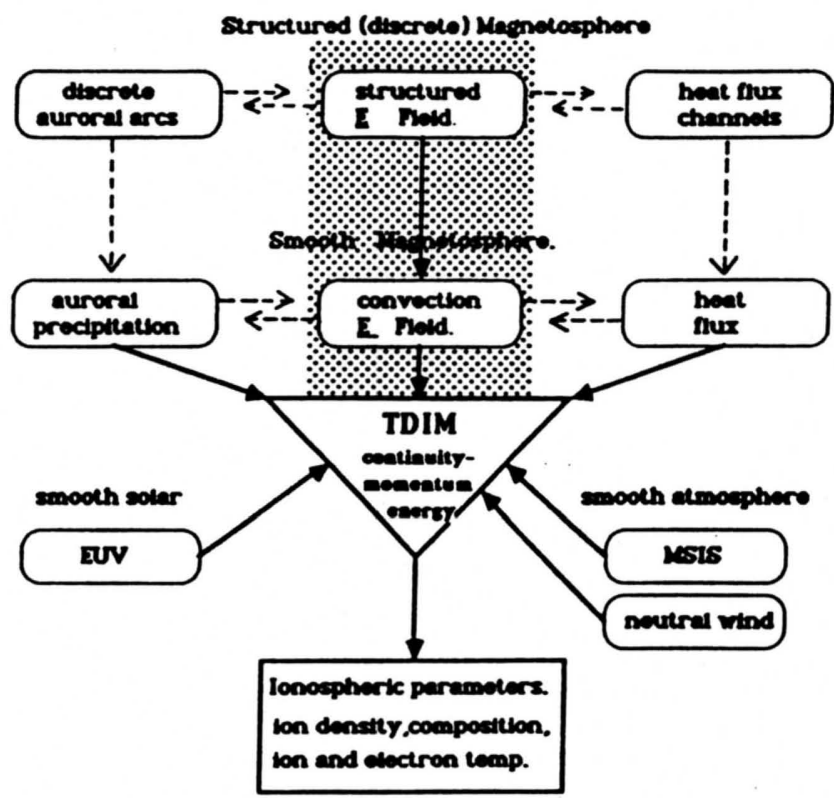

Fig. 11. Schematic of how structure mechanisms affect the $F$ region as represented by a physical model. The shading indicates the scope of this study. Dashed lines indicate links in the coupling processes which cannot be physically described at this time.

tern. Four sets of electric field structures were used to generate convection models: (1) zero case, structures of zero electric field which corresponds to the conventional two-cell convection pattern; (2) case $A$, a total of 45 structures whose mean electric potential drop was $5 \mathrm{kV}$; (3) case $B$, the identical 45 structures in case $A$ but whose mean electric potential drop was increased to $12 \mathrm{kV}$; (4) reverse polarity case, identical to case B but with the polarity of the electric field in the central region being changed from dawn-dusk to dusk-dawn.

For each of these four convection models the USU-TDIM was run for a group of eight transpolar plasma flux tubes. The plasma parameters associated with these runs were contrasted and the following results were obtained:

1. The overall convection process with the inclusion of the electric field structures leads to different trajectories and transpolar crossing times. This implies that prior to entering the polar cap the initial plasma characteristics are different. Alone this difference can produce differences of factors of 4 in the overall $N_{m} F_{2}$ values. Initial conditions depend heavily on universal time and location outside the polar cap especially during winter and equinox when the terminator's location is important. Consequently, an understanding of plasma past history outside the polar cap would also be critical.

2. With enhanced electric fields being present in the polar cap for models case A, case B, and case B reverse polarity, the plasma polar cap transit times are generally reduced. Contrary to simple argument, this faster transit time for a dark polar cap (no plasma production sources) does not yield higher densities.

3. The electric field structures lead to enhanced $T_{i}$, which in turn lead to enhanced $\mathrm{O}^{+}$loss via the reaction $\mathrm{O}^{+} \rightarrow \mathrm{NO}^{+}$. This process is the dominant $N_{m} F_{2}$ mechanism. For the case B model it is responsible for factors of 4 lower $N_{m} F_{2}$ after the polar cap traversal.

4. The final $N_{m} F_{2}$ values for cases A and B are generally lower than the zeroth case. For case $A$ the final densities seem randomly lower than the corresponding zero case. In contrast, for case $B$ the final densities appear to be systematically lower than the corresponding zero case (see Table 4). This difference between case A and case B may not be important. Follow up studies will have to explore whether this difference is statistically meaningful.

5. $H_{m} F_{2}$ experiences a variety of responses to both induced vertical drifts and rapid changes in composition and temperature. However, $h_{m} F_{2}$ and $N_{m} F_{2}$ are not systematically correlated. In general, $h_{m} F_{2}$ is increased in the electric field structures by tens to hundreds of kilometers, depending on the electric field strength.

6. The $F$ region peak and topside respond relatively slowly to small scale structures and, indeed, $N_{m} F_{2}$ responds by only a few percent changes to sudden changes in the electric field. The integrated effect of the larger electric field (i.e., time spent in such structures) leads to systematic decrease in the $F$ layer.

7. In contrast the topside molecular ion density, specifically $\mathrm{NO}^{+}$, responds dramatically to sudden increases in the electric field. The $\mathrm{NO}^{+}$density at $800 \mathrm{~km}$ can increase by over 3 orders of magnitude in tens of seconds. This phenomena successfully pumps molecular ions into the bottom of the magnetosphere.

Because of the large number of parameters used to define the structures, and then using random number generators to select these parameters, this study of eight trajectories per case is not extensive enough to be treated as a Monte Carlo simulation. Hence the results cannot be treated as statistically significant, but are merely indicative of the stochastic trends associated with the electric field structures.

The overall impact of these electric field structures on the polar ionosphere is probably non-negligible. These structures exist during periods of IMF $\boldsymbol{B}_{\boldsymbol{z}}=\mathbf{0}$ or positive, however, how representative the model structures are, still needs further investigation. Without ascertaining the validity of the input structures, the conclusions can only give an indication of the possible types of ionospheric consequences. No theoretical description is available to extend the in-situ satellite data sets into a horizontal two-dimensional picture or define their temporal characteristics. In this study, a two-dimensional, two-cell model has been adopted with the assumption that these structures are stable for the length of time it takes a plasma flux tube to cross it. For the eight test trajectories, the average transit times through the individual structures range from 50 to $300 \mathrm{~s}$. This study therefore assumes that the duration of an electric field structure is between 1 and $5 \mathrm{~min}$.

These test trajectories have been chosen such that they all follow different paths but arrive at the same time at their respective end points. If we had attempted to study the UT dependences of each trajectory, then the steady electric field structures would not be applicable. For studies in which a time evolution of the polar ionosphere is to be studied, the lifetime question of each structure must be addressed. Given that the structure model is not entirely unreasonable, it appears that the structures contribute to ionospheric processes in several ways; reduce $N_{m} F_{2}$, modify $h_{m} F_{2}$, locally enhance $T_{i}$, pump molecular ions to high altitudes, and lead to large-scale structuring in $N_{m} F_{2}$. The degree to which each of these ionosphere modifications depends upon the adopted structure model is very different. In describing the final $N_{m} F_{2}$ variations, it is evident that two components affect it; first there is change in the initial conditions due to the different trajectories and transit times and, second, while in the vicinity of each electric field structure, drastic $N_{m} F_{2}$ changes can occur. Hence it is not only necessary to know the electric field 
details of each structure, but also to know how the structures are distributed and coupled. For example, by just changing the distribution geometry of the structures within the polar cap, which leads to different trajectories and, hence, transit times, would lead to modified initial densities and, consequently, final densities. Alternatively, keeping the distribution the same but changing the size or characteristics of the electric field would also lead to local changes in $N_{m} F_{2}$, causing the final densities to change. Other parameters such as $T_{i}$ or the composition, where the time constants are short compared to the transpolar crossing times, would be more strongly dependent upon the details of the individual structures rather than the integrated effect of many structures.

The above results are derived from a theoretical study of how electric field structures modify $F$ region plasma parameters. The study on its own is not a polar cap $F$ region study. It only indicates quantitatively the integrated effect of multiple electric field structures upon the ionospheric $N_{m} F_{2}, h_{m} F_{2}$ and composition. These electric field structures are themselves only weakly representative of polar cap structures observed during periods of zero to northward IMF $B_{z}$ conditions. In order for this type of study to be directly related to the polar cap ionosphere, significantly improved understanding of the "structure mechanism" must be obtained. Schematically, Figure 11 shows the various processes which probably contribute to polar cap ionospheric structures. The strengths of the various couplings between the magnetospheric inputs are at this time relatively unknown. Several previous studies have been carried out to investigate how these other structure processes affect the $F$ region; discrete auroral precipitation [Robinson et al., 1985; Sojka and Schunk, 1986]; neutral winds [Sojka and Schunk, 1985b]. In the schematic, the shaded region indicates the subset of magnetospheric inputs which have been studied in this paper for three adopted structure models. None of the couplings have been included. Significant advancement in our understanding, either empirically or theoretically, would be required before such couplings can be included in this type of study. At the present time, extensive data bases, such as that of the Dynamics Explorer (DE) project, would be ideal for such empirical studies. Based upon the $F$ region modifications inferred from this study, it is very important that this type of modeling be extended to include these couplings with the other magnetospheric-ionosphericthermospheric inputs.

\section{APPENDIX}

The electric field structure inside the polar cap is defined as a sub-region whose center is at a MLT and magnetic latitude $\left(\mathrm{MLT}_{0}, \mathrm{DLAT}_{0}\right)$. This location is taken to be the new origin. Cartesian coordinates are constructed as follows: $+x$ direction is antisunward while the $+y$ direction is dawnward parallel to the dawn meridian. A given location is then defined by $\left(x_{p}, y_{p}\right)$ which can be transformed to a spherical polar latitude and longitude about this origin as follows ( $x_{p}$ and $y_{p}$ are in degrees of latitude at $300 \mathrm{~km}$ ):

$$
\begin{gathered}
\text { lat }=90^{\circ}-\left(x_{p}^{2}+y_{p}^{2}\right)^{1 / 2} \\
\text { long }=\tan ^{-1}\left(y_{p} / x_{p}\right)
\end{gathered}
$$

An Eulerian transformation can then be used to express this

location in magnetic latitude-MLT coordinates.

Centered on the displaced origin $\left(x_{0}, y_{0}\right)$ is an inner region of uniform electric field; this region being elliptical in shape. Out- side this inner region the electric field diminishes to zero at the outer boundary; again an ellipse. Inside the inner region the electric field is orientated along the dawn-dusk (or vice versa) direction. The inner region is extended in the noon-midnight direction. Figure $1 a$ shows the orientation of these two boundaries. The inner region's semiminor axis $\left(r_{0}\right)$ lies parallel to the dawn-dusk direction. A parameter $\left(f_{\text {in }}\right)$ is defined to be the ratio of the semimajor $\left(r_{m}\right)$ to semiminor axis $\left(f_{\text {in }}=r_{m} / r_{0}\right)$. Inside this central region the electric field is constant and is defined by a total potential drop of $\phi_{0} \mathrm{kV}$. The electric potential $\phi\left(x_{p}, y_{p}\right)$ is then chosen to be zero along the $y_{p}=0$ line and hence;

$$
\phi\left(x_{p}, y_{p}\right)=\frac{y_{p} \phi_{0}}{2 r_{0}}
$$

An outer ellipse defines the region beyond which the electric field for this structure is zero. This outer ellipse has the same orientation and eccentricity as the inner ellipse. Its semiminor axis $\left(r_{2}\right)$ is given by $r_{2}=r_{0} f_{\text {out }}$. Any point lying between the two ellipses can be regarded as being at a radial distance $r$ from the origin. This radius vector intersects the inner ellipse at $r_{b}$ and the outer ellipse at $r_{\text {out }}$. Equation (A3) can then be used to determine the potential at $r_{b}, \phi_{b}$ and by definition the potential at $r_{\text {out }}=0$. Since the electric field diminishes linearly between $r_{b}$ and $r_{\text {out }}$, the electric potential between these two boundaries $\phi(r)$ is given by

$$
\phi(r)=\phi_{b}\left(\frac{r_{\text {out }}-r}{r_{\text {out }}-r_{b}}\right)^{2}
$$

Acknowledgments. This research was supported by NASA grants NAG-5-748 and NAGW-77 and AFOSR contract F49620-86-C-0109 to Utah State University.

The Editor thanks S. Quegan and another referee for their assistance in evaluating this paper.

\section{REFERENCES}

Burke, W. J., M. C. Kelley, R. C. Sagalyn, M. Smiddy, and S. T. Lai, Polar cap electric field structures with a northward interplanetary field, Geophys. Res. Lett., 6, 21-24, 1979.

Conrad, J. R., and R. W. Schunk, Diffusion and heat flow equations with allowance for large temperature differences between interacting species, J. Geophys. Res., 84, 811-822, 1979.

Frank, L. A., et al., The theta aurora, J. Geophys. Res., 91, 3177-3224, 1986.

Heelis, R. A., and W. B. Hanson, High-latitude ion convection in the nighttime $F$ region, J. Geophys. Res., 85, 1995-2002, 1980.

Heelis, R. A., J. K. Lowell, and R. W. Spiro, A model of the highlatitude ionospheric convection pattern, J. Geophys. Res., 87, 63396345, 1982.

Heelis, R. A., P. H. Reiff, J. D. Winningham, and W. B. Hanson, Ionospheric convection signatures observed by DE-2 during northward interplanetary magnetic field, J. Geophys. Res., 91, 5817-5830, 1986.

Heppner, J. P., Polar cap electric field distributions related to the interplanetary magnetic field direction, J. Geophys. Res., 77, 48774887, 1972.

Heppner, J. P., High latitude electric fields and the modulations related to interplanetary magnetic field parameters, Radio Sci., 8, 933-948, 1973.

Heppner, J. P., Empirical models of high-latitude electric fields, J. Geophys. Res., 82, 1115-1125, 1977.

Kan, J. R., and W. J. Burke, A theoretical model of polar cap auroral arcs, J. Geophys. Res., 90, 4171-4177, 1985.

Kelly, J. D., and J. F. Vickrey, $F$ region ionospheric structures associated with antisunward flow near the dayside polar cusp, Geophys. Res. Lett., 9, 907-910, 1984.

Kintner, P. M., and C. E. Seyler, The status of observations and theory of high latitude ionospheric and magnetospheric plasma turbulence, Space Sci. Rev., 41, 91-129, 1985.

Knudsen, W. C., P. M. Banks, J. D. Winningham, and D. M. Klumpar, 
Numerical model of the convecting $F$ ionosphere at high-latitudes, J. Geophys. Res., 82, 4784-4792, 197;

Koffman, W., and V. B. Wickwar, Very high electron temperatures in the daytime $F$ region at Sondrestrom, Geophys. Res. Lett., 9, 919922, 1984.

Quegan, S., G. J. Bailey, R. J. Moffett, R. A. Heelis, T. J. FullerRowell, D. Rees, and R. W. Spiro, A theoretical study of the distribution of ionization in the higher latitude ionosphere and the plasmasphere: First results on the mid-latitude trough and the light ion trough, J. Atmos. Terr. Phys., 44, 619-640, 1982.

Robinson, R. M., R. T. Tsunoda, J. F. Vickery, and L. Guerin, Sources of $F$ region ionization enhancements in the nighttime auroral zone, J. Geophys. Res., 90, 7533-7546, 1985.

Roble, R. G., R. E. Dickinson, and E. C. Ridley, Global circulation and temperature structure of the thermosphere with high-latitude plasma convection, J. Geophys. Res., 87, 1599-1614, 1982.

Schunk, R. W., and W. J. Raitt, Atomic nitrogen and oxygen ions in the daytime high-latitude $F$ region, J. Geophys. Res., 85, 1255-1272, 1980.

Schunk, R. W., and J. J. Sojka, Ion temperature variations in the daytime high-latitude $F$ region, $J$. Geophys. Res., 87, 5169-5183, $1982 a$.

Schunk, R. W., and J. J. Sojka, Ionospheric hot spot at high latitudes, Geophys. Res. Lett., 9, 1045-1048, 1982 b.

Schunk, R. W., and J. C. G. Walker, Theoretical ion densities in the lower ionosphere, Planet. Space Sci., 21, 1875-1896, 1973.

Schunk, R. W., W. J. Raitt, and P. M. Banks, Effect of electric fields on the daytime high-latitude $E$ and $F$ regions, J. Geophys. Res., 80, 3121-3130, 1975.

Schunk, R. W., P. M. Banks, and W. J. Raitt, Effects of electric fields and other processes upon the nighttime high latitude $F$ layer, $J$. Geophys. Res., 81, 3271-3282, 1976.

Smiddy, M., W. J. Burke, M. C. Kelley, N. A. Saflekos, M. S. Gussenhoven, D. A. Hardy, and F. J. Rich, Effects of high-latitude conductivity on observed convection electric fields and Birkeland currents, $J$. Geophys. Res., 85, 6811-6818, 1980.
Sojka, J. J., and R. W. Schunk, A theoretical study of the global $F$ region for June solstice, solar maximum, and low magnetic activity, J. Geophys. Res., 90, 5285-5298, 1985a.

Sojka, J. J., and R. W. Schunk, Theoretical study of anomalously high $F$ region peak altitudes in the polar ionosphere, J. Geophys. Res., 90 , $7525-7532,1985 b$.

Sojka, J. J., and R. W. Schunk, A theoretical study of the production and decay of localized electron density enhancements in the polar ionosphere, J. Geophys. Res., 91, 3245-3253, 1986.

Sojka, J. J., W. J. Raitt, and R. W. Schunk, A theoretical study of the high-latitude winter $F$ region at solar minimum for low magnetic activity, J. Geophys. Res., 86, 609-621, 1981a.

Sojka, J. J., W. J. Raitt, and R. W. Schunk, Theoretical predictions for ion composition in the high-latitude winter $F$ region for solar minimum and low magnetic activity, J. Geophys. Res., 86, 2206-2216, $1981 b$.

Spiro, R. W., P. H. Reiff, and L. J. Maher, Precipitating electron energy flux and auroral zone conductances: An empirical model, J. Geophys. Res., 87, 8215-8227, 1982.

van Eyken, A. P., H. Rishbeth, D. M. Willis, and S. W. H. Cowley, Initial EISCAT observations of plasma convection at invariant latitudes $70^{\circ}-77^{\circ}, \mathrm{J}$. Atmos. Terr. Phys., 46, 635-641, 1984.

Volland, H., A semiempirical model of large-scale magnetospheric electric fields, J. Geophys. Res., 78, 171-180, 1973.

Volland, H., Models of global electric fields within the magnetosphere, Ann. Geophys., 31, 154-174, 1975.

Watkins, B. J., A numerical computer investigation of the polar $F$ region ionosphere, Planet. Space Sci., 26, 559-569, 1978.

R. W. Schunk and J. J. Sojka, Center for Atmospheric and Space Sciences, UMC 4405, Utah State University, Logan, UT 84322.

(Received March 12, 1987;

revised October 7, 1987;

accepted September 22, 1987.) 